\title{
Modelling coral reef biodiversity and habitat destruction
}

\author{
Lewi Stone*, Efrat Eilam, Avigdor Abelson, Micha Ilan \\ Department of Zoology, Tel Aviv University, Ramat Aviv 69978, Tel Aviv, Israel
}

\begin{abstract}
World wide coral reef decline has now been well documented, but the actual dynamics of this disturbing phenomenon are still far from understood. In this note we describe a simple spatial mathematical model that attempts to capture some of the important ecological processes - including colonization, mortality and competition for space-all of which govern questions of species coexistence in coral communities. The model is then extended to determine what might happen to a community if a proportion of the spatial landscape is destroyed and can no longer be occupied. We examine the species extinction debt incurred as habitat destruction increases and attempt to predict the characteristics of those species which are at greatest risk. To gain even further insights, the results of the model are compared with field-data from the reef flats of Eilat, Israel, as found in Loya's (1976; Ecology 57:278-289) classic study of community structure at reef sites damaged by chronic oil pollution.
\end{abstract}

KEY WORDS: Coral reef $\cdot$ Habitat destruction $\cdot$ Spatial model Extinction Recruitment.Competition-Oil-pollution

Coral reefs, the most complex and diverse of all aquatic ecosystems, are currently facing widespread devastation. Wilkinson (1993) warned that some $70 \%$ of the world's reefs will be completely destroyed over the next 40 yr and massive species extinctions are to be expected if the process is not soon reversed. The recent model of Tilman et al. (1994) (henceforth referred to as TMLN) becomes of particular relevance, for it predicts the species extinction debt in ecosystems as habitat destruction $(D)$ increases. Here we modify TMLN and test it with field data from the reef flats in Eilat, Israel. The following note is intended to focus more on the ecological implications of our findings. Complete details concerning the more technical theoretical and mathematical aspects of our work will be presented in a companion paper (Stone 1995).

The spatial model described by TMLN and also used here, was originally designed by Hastings (1980) for application to coral reef communities. In such commu-

•E-mail: lewi@lanina.tau.ac.il nities, species have been understood to interact in a hierarchical web of competitive interactions. In all of the above models, a set of $\mathrm{N}$ species colonize and compete for a large number of patches according to a ranked hierarchy; species 1 is taken to be the best competitor for space, while species $\mathrm{N}$ is the weakest. To enhance coexistence, the model incorporates a 'competition-colonization' trade-off (Tilman 1994) such that the better competitors are poor colonizers while the good colonizers are weak competitors. Because individuals are mortal, species are constantly losing patches by the usual death process. This in turn provides a continuous source of newly opened patches for which species compete. On average, the weaker competitors are able to colonize vacant patches more rapidly. This is how the trade-off between competition and colonization helps prevent the best competitor from eventually taking over all possible space.

In the terminology of the present note, we let $p_{t}$ denote the proportion of the total number of patches on which species $i$ resides, which is also loosely referred to here as the species' abundance. The colonization potential of the $i$ th species is taken to be $c_{i}$, while its mortality rate is $m_{i}$. Following TMLN, we assume that a proportion $D$ of sites, where $0 \leq D \leq 1$, are permanently destroyed and can never be colonized. Ultimately we seek to determine the effects of habitat destruction on a model coral reef system.

The spatial growth of the $i$ th species is modelled by the differential equation (Hastings 1980, TMLN):

$$
\frac{\mathrm{d} p_{i}}{\mathrm{~d} t}=c_{1} p_{i}\left(1-D-\sum_{j=1}^{i} p_{j}\right)-m_{1} p_{1}-\sum_{j=1}^{i-1} c_{i} p_{i} p_{i}
$$

There are 3 terms on the right hand side of the equation.

(1) The first term represents the colonization process. Because there is a competitive hierarchy, a species is only free to invade sites which are empty or on which species lower in the hierarchy reside. Keeping in mind that a proportion $D$ of sites are unavailable for colo- 
nization because of habitat destruction, the fraction of available sites for the $i$ th species to colonize is thus

$$
\left(1-D-\sum_{j=1}^{i} p_{j}\right)
$$

The rate at which species $i$ randomly colonizes available space is the product of this fraction, the species' colonization potential $c_{2}$, and its present abundance $p_{1}$. [Note that the per capita recruitment rate (found from examining $\frac{1}{p_{i}} \cdot \frac{\mathrm{d} p_{i}}{\mathrm{~d} t}$ ) is density dependent since it is the product of the fraction of available sites and $c_{1}$.

(2) The second term $\left(m, p_{i}\right)$ is the rate of loss of occupied patches by a species because of mortality which is, technically speaking, density independent.

(3) The last term models the effects of competition. In this system, the $i$ th species can only be outcompeted and thus lose space to species that are higher in the hierarchy (specifically species $1,2, \ldots, i-1$ ). The rate at which species $i$ loses patches to the better competitor species $j(j<i)$, is approximated by the non-linear Lotka-Volterra term $c_{1} p_{i} p_{j}$. This term reflects not only the colonization rate of species $j$, but also the amount of space (number of patches) it already occupies.

The major advantage of this Lotka-Volterra formulation of hierarchical competition is that it greatly facilitates mathematical analysis thereby providing a first step in understanding theoretically the underlying community dynamics. However, some of the simplifying model assumptions may be at the expense of ecological realism. For example, it is well known that corals generally interact with their nearby neighbours and rarely at random as assumed by the Lotka-Volterra approach. (For the model defined by Eq. (1), even this may not be a serious problem since the colonization process effectively ensures that neighbours will in any case be random). Furthermore, strict linear dominance hierarchics amongst corals are generally realistic when only 1 mechanism of competition is being considered, but since corals may have many ways in which they compete, the linear hierarchy transforms into a more complex network. Nevertheless, the model used here appears to have some robustness to structural changes and its characteristic behaviour is remarkably close to that found in similarly formulated cellular automata neighbourhood models (see TMLN) as well as in adaptions for which the assumption of strict hierarchy is loosened (Tilman pers. comm., Tilman \& Lehman unpubl.).

Because Eq. (1) necessarily possesses a unique globally stable equilibrium (Hastings 1980, Stone 1995), the model may be analysed by following the time-course of all populations until the steady-state configuration is reached with $\mathrm{d} p, / \mathrm{d} t=0$ for all species. This 'spatial steady-state' is different to the usual population equilibrium as commonly referred to in the ecological liter- ature. Globally, over the entire spatial landscape an equilibrium is maintained, but if observed on more local scales (e.g. clusters of nearby patches) populations continuously fluctuate. Interestingly, by analysing stable spatial. steady-state configurations arising from equations similar to Eq. (1), Hastings (1980) was able to successfully simulate Connell's non-equilibrium intermediate disturbance theorem.

TMLN analysed the above model in their study of the effects of habitat destruction on forest ecosytems where it was assumed that the top competitor is the most abundant species. Two very important outcomes of their study can be simply stated. (1) Surprisingly, as habitat destruction is increased, it is the most abundant species (i.e. the top competitor) that is the most fragile and is the first to go extinct. (2) Species extinctions are time delayed and may occur many decades after habitat is actually destroyed.

We checked the possibility that coral reef ecosystems may have similar dynamics to those predicted by the model for forest communities by first examining existing field research at the reef flats in Eilat, Israel. Loya (1976) surveyed 2 sites of the Eilat reef 1 yr before, and 3 yr after, an extraordinary and severe low-tide event in 1970 . The low tide led to a catastrophic mass mortality of corals but without damaging the actual habitat itself. Soon after his first survey, major oil spills followed by chronic pollution seriously damaged one of the sites. Loya then compared the build up and assembly of the coral communities at both sites finding that while the unpoliuted $(D=0)$ control site rapidly returned very close to its former configuration in a matter of 3 yr (Fig. 1b), the chronically polluted $(D>0)$ site never recovered, suffered numerous species extinctions and showed a radically altered community structure (Fig. 1a). More recent field surveys confirmed a qualitatively similar ecological scenario (Loya 1990). All of these findings so far stand in complete accord with the predictions of TMLN.

We used Loya's data to calibrate the TMLN model but first noted major differences in basic assumptions. For the Eilat reefs, Loya (1976) painstakingly documented how 'the commonest species ... had the highest rates of recruitment': an observation that stands in sharp contrast to TMLN. Some of the most common species that were also found to be good colonizers include Stylophora pistillata, Cyphastrea microphthalma and Millepora dichotoma. According to Loya (1976) (although there may be exceptions), 'opportunistic species,' of which the above are considered examples, 'have opted for high reproductive rates, short life spans, large population sizes, wide physiological tolerances, broad dispersal abilities, density independent mortalities, and poor competitive abilities... Most of these characteristics are exhibited by 


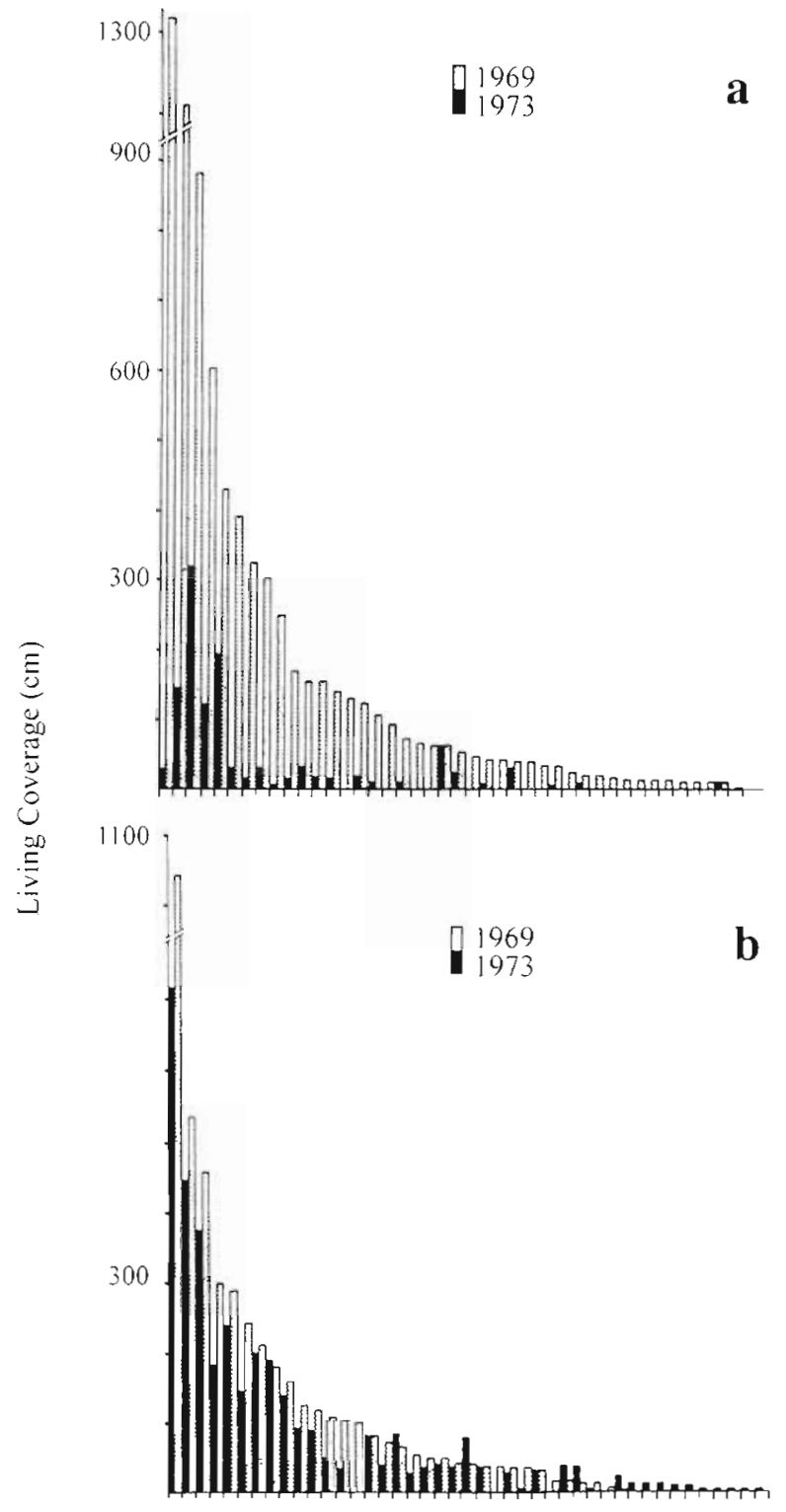

\section{Species}

Fig. 1. A comparison between the total living coverage of each coral species in 1969 and 1973 in Eilat. (a) Polluted reef $(D>0)_{i}$ (b) unpolluted reef $(D=0)$. The living coverage of each coral species is represented by a vertical bar, and species are ordered from left to right after ranking them according to coverage. The graphs are modified from Loya (1976) where a species listing is given. According to data from Abelson (1987) and Loya (1976), the better colonizers are found on the left side of horizontal axes (i.e. with highest coverage) while the weaker colonizers are to be found on the right side (i.e. with lowest coverage). The competition-colonization trade-off posits that the least aggressive species are to be found on the left while the most aggressive are on the right side of the horizontal axes (see text for discussion). This latter relationship is difficult to check in practice largely because of the many problems in obtaining a true measure of aggressiveness. Abelson's (1987) study suggests this general trend for these reefs although there are several exceptions. The above graphs are reproduced with the permission of the Ecological Society of America the most common coral species of the reef flat.' We therefore modified the model to accomodate this difference by giving species $N$, the best colonizer, the highest population abundance while species 1 , the weakest colonizer, was assigned the lowest population abundance. In mathematical terms, we set the population of species $i$ as $p_{1} \approx\left[1+0.05(\mathrm{~N}-i+1)^{2}\right]^{-1}$, which approximates the actual data (Fig. 1) and ensures that: $p_{1}<p_{2}<p_{3}<\ldots<p_{N}$. Both coral communities comprised close to 40 species and it seemed representative to examine model systems taking $\mathrm{N}=40$.

The true impact of interspecific competition for space at the community level is controversial in reef systems (Lang \& Chornensky 1990), and appears to be of minimal consequence in Eilat (Abelson 1987). To help filter out the role of competition in the model we assumed species' recruitment or colonization potentials $\left(c_{i}\right)$ were largely responsible for (Loya 1976, Abelson 1987), and proportional to, the population abundances i.e. $c_{i} \approx p_{i}$. (Note that in theory, it would be more accurate to take colonization rates as proportional to the actual number of colonies-here, species living coverage divided by their growth rates. In the case of Loya's data, however, the distribution of living coverage and the distribution of the number of colonies are extremely similar so that this distinction makes litthe practical difference). The model's built-in competition-colonization trade-off indirectly produces a fixed inverse relationship between competitive ability and abundance; an assumption that is not unreasonable in some coral reefs (Loya 1976, Jackson \& Hughes 1985). The best competitor is not only the weakest colonizer, but also has the lowest population abundance. Both Loya's (1976) and Abelson's (1987) findings confirm that many of the aggressive corals in Eilat's reef flats are of relatively low abundances, although there are some exceptions. Because of the many difficulties involved in determining a true index of aggressiveness, in practice no simple relationship between abundance and aggression has been determined for all species as yet, although the expected trend is discernible. Several typical aggressive corals of low abundance include Galaxea fascicularis, Goniopora lichen, Platygyra subdentata and Platygyra rustica. Unlike the TMLN model, mortality rates $\left(m_{i}\right)$ could not be assumed constant for each species and calculations (Tilman 1994, his Eq. 8) yield in general $m_{1}<m_{2}<m_{3}$ $\ldots<m_{N \text { r }}$ so that superior colonizers have the highest rates of mortality. The above scheme in terms of ranking in hierarchy, abundance, colonization and mortality is well known for numerous coral communities (Loya 1976, Jackson \& Hughes 1985, Abelson 1987).

With the parameters $m_{1}$ and $c_{1}$ now all determined, the model was examined for many different values of habitat destruction $D$. In doing so, we immediately 


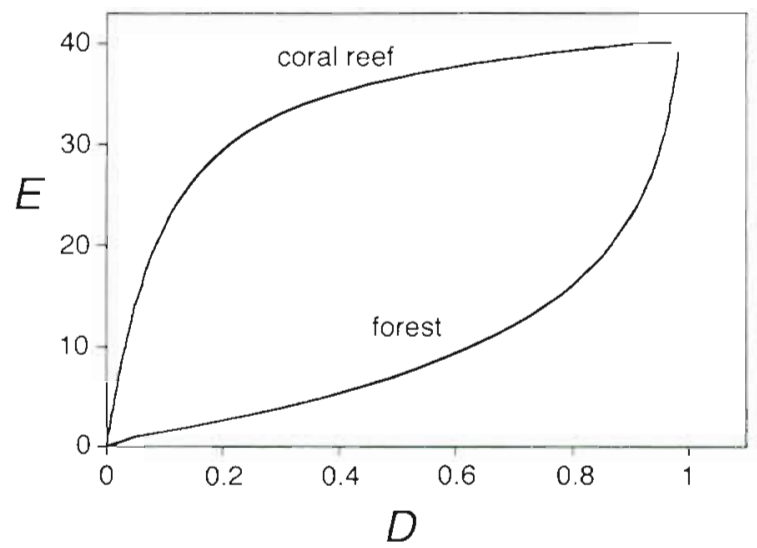

Fig. 2. Calculated extinction debt $E_{1}$ which represents the number of species that eventually become extinct as a function of habitat destruction $D$. Results are given for both the forest model [details in TMLN $(\mathrm{q}=0.05)$ ] and coral reef model (simulations according to scheme in text) with $\mathrm{N}=40$ species

found that the modified model leads to a conclusion that directly contradicts one of the major findings of TMLN. The latter authors predict that common species should be the first to go extinct in the presence of habitat destruction. Instead we found that habitat destruction drives the weakest colonizers, with low abundance levels, to extinction first, while the best colonizers with high abundances survive, just as the real data demonstrates (Fig. 1a). This occurs because the high colonization ability of species lowest in the hierarchy indirectly endows them with considerable 'competitive edge' over their more aggressive rivals. The prediction of TMLN that the top competitor is the most fragile remains intact. The model findings agree with the real data: the large drop in species numbers at the site damaged by oil pollution (Fig. 1a) was largely due to the inability of aggressive competitors such as Galaxea fascicularis, Goniopora lichen, Platygyra subdentata and Platygyra rustica (all of low abundances) to survive or reestablish themselves; at the undamaged site (Fig. 1b) there was no extinction debt whatsoever.

On a more worrying note, the modified model indicates that only very mild habitat destruction may lead to a large extinction debt. As Fig. 2 shows, damage to $10 \%$ of the habitat led to the extermination of some $50 \%$ of existing species. If the populations were simultaneously depressed by a simulated low-tide event these extinctions could be rapid-even immediate. Low-tide events thus serve to speed up the time-delay to extinction for endangered species, as simulations have shown.

The extinctions should be considered local, and it is conceivable that rather than permanently disappear- ing, some of these species may later be externally recruited, only to perish once again after failing to establish themselves. Although Eq. (1) describes a closed system, it also has some capacity to represent an open system in which pulses of external recruitment arrive infrequently. In some cases, such recruitment pulses can act to boost the population levels of endangered species and prevent them from ever equilibriating to extinction. To understand in more detail some of the other complex properties of open systems would necessitate adding immigration terms to Eq. (1), which although beyond the scope of the present analysis, is certainly worthy of future exploration.

The extinction rates noted here are far larger than estimates obtained from the TMLN model, simply because in our simulations a significant proportion of species have the combined disadvantage of low abundance and poor recolonization ability, making them extremely vulnerable to disturbance and habitat destruction. While the TLMN forest model does not allow for species with this twin combination of disadvantageous traits, field data suggests that it is commonplace for corals and significantly increases extinction risk. Results such as these indicate the need to treat with seriousness Wilkinson's (1993) distressing predictions concerning future devastation and biodiversity decline in the world's coral reefs.

\section{LITERATURE CITED}

Abelson A (1987) Aggression amongst stony corals: is it a competition for space? MSc thesis, Tel Aviv University

Hastings A (1980) Disturbance, coexistence, history, and competition for space. Theor Pop Biol 14:380-395

Jackson JBC, Hughes TP (1985) Adaptive strategies of coral reef invertebrates. Am Sci. 73:265-274

Lang JC, Chornensky EA (1990) Competition between scleractinian reef corals - a review of mechanisms and effects. In: Dubinsky $Z$ (ed) Ecosystems of the world: coral reefs Elsevier, Amsterdam, p 209-250

Loya Y (1976) Recolonization of Red Sea corals affected by natural catastrophes and man-made perturbations. Ecology $57: 278-289$

Loya Y (1990) Changes in a Red Sea coral community structure: a long-term case history study. In: Woodwell GM (ed) The earth in transition. Cambridge University Press, Cambridge, p 369-384

Stone L (1995) Biodiversity and habitat destruction: a comparative study of model forest and coral reef ecosystems. Proc Royal Soc B 261:381-388

Tilman D (1994) Competition and biodiversity in spatially structured habitats. Ecology 75:2-16

Tilman D, May RM, Lehman CL, Nowak MA (1994) Habitat destruction and the extinction debt. Nature 371:65-66

Wilkinson CR (1993) Coral reefs of the world are facing widespread devastation: can we prevent this through sustainable management practices? Proc 7 th Int Symp Coral Reef $1: 11-21$

Manuscript first received: April 26, 1995

Revised version accepted: October 30, 1995 